\title{
Research on electrical automation teaching reform based on artificial intelligence and virtual simulation technology
}

\author{
Yan $\mathrm{Huo}^{1}$, and Jie $\mathrm{Hou}^{2}$,* \\ ${ }^{1}$ School of Information Engineering, Shenyang University, Shenyang, China \\ ${ }^{2}$ School of Automation, Chongqing University of of Posts and Telecommunications, Chongqing, China
}

\begin{abstract}
With the rapid development of modern science and technology, electrical automation becomes more and more important in the development of manufacturing industry. In the actual production activities, the application of artificial intelligent and virtual simulation technology in electrical engineering not only improves automation operation level, and reduces the cost and the error of manual operation, but also profits reasonable allocation for enterprise resources. In recent years, the demand for talents with new automation technology is increasing. Therefore, an innovative model of automation teaching reform based on information technology including artificial intelligent and virtual simulation technology is established to constitute an oriented training system from aspects of teaching and learning. Information technology plays an increasingly important role in the field of engineering training. The effective combination of electrical product, virtual simulation and intelligent technology can enhance the application value of teaching method and meet the practical needs of future engineering construction.
\end{abstract}

\section{Introduction}

In the electrical engineering field, the overall mode mainly includes control module, operation module, and information processing and application module. Electrical automation plays an important role in manufacturing industry, which is a main processing part of the automation system affecting the overall mode of modern production [1-2]. Nowadays the current situation and development trend of electrical engineering automation is being explored by adjusting and optimizing complex engineering problems in manufacturing processes, in order to achieve the purpose of automatic control and monitoring.

In recent years, the development of technical society is inseparable from the support of information technologies. For example, the effective integration of information technology and automation control system or sensor equipment can further strengthen production function. In the process of industrial development, the integration of multimedia technology, and computer network method can improve the operation efficiency and control level of automation system to a certain extent. Intelligent production has been developed without human intervention to improve the flexibility of production and resource utilization. The new model covers intelligent design [3], intelligent process planning, intelligent measurement, and intelligent decision-making etc. How to cultivate the new style talents of electrical automation with strong practical ability and innovation ability is the key to the teaching model reform with artificial intelligent and virtual simulation technology [4].
In the teaching reform, with the help of electrical training teaching simulation platforms, the electrical control schematic diagram and program show the electrical control process and electrical components, stimulating student learning, to improve the teaching efficiency.

With the development of information technology, virtual experiment teaching enriches the traditional experiment teaching [5]. It cannot only replace some traditional experiments, but also avoid the limitations and disadvantages of various practical factors in traditional experiments, and solve many problems in experiment teaching. By using computing network technology, virtual reality technology and multimedia technology, we can simulate most of advanced and complex experimental equipment, reduce the experimental cost and security risk, provide free and open experimental environment for learners, and improve learners' autonomy and innovation. Therefore, the new methods can promote the reform of teaching mode and the innovation of education concept.

\section{Traditional mode analysis}

There are several restriction factors of carrying out teaching activities for electrical automation under the traditional mode. There are some elements in the experimental teaching model as shown in Table 1. In terms of objective conditions, the main restriction factors can be roughly summarized as follows: time is constrained in the designated class time; place is constrained in the designated experimental place;

* Corresponding author: jiehou.ph@gmail.com 
experimental tools are constrained by hardware equipment or materials; experimental environment is constrained by buildings, equipment and other local environmental conditions. Because the structure of the electrical control system is not very simple, so the experimental cost will not be too low, and the experimental equipment in the laboratory is not easy to control.

Table 1. Model elements.

\begin{tabular}{|c|c|}
\hline Elements & Statement \\
\hline Time & Designated class time \\
\hline Place & Designated experimental place \\
\hline Tool & Hardware or materials \\
\hline Environment & Buildings and conditions \\
\hline
\end{tabular}

As far as the teaching mode is concerned, the restriction factors are mainly reflected in the traditional teaching methods. The traditional teaching method often uses the unchanged forms of teachers' explanation and students' listening. Then students are not good at mastering the designing or operation essence of the electrical control system well. It is not easy to solve the practical operation problems in the teaching processes of electrical automation courses.

In the traditional experimental teaching mode, the teaching method is single. Teachers explain and demonstrate the experimental content in class, and students simply imitate the experimental content to verify the correctness of the experimental results. However, students' initiative and independent innovation ability have not been inspired and cultivated. This old teaching mode is easy to produce the students with high score and low ability. So the traditional mode can not meet the talent demand of high-tech enterprises for the current development of automation industry.

It is well known that automation teaching may be supplemented by using certain new technology. For example, some software training systems can meet most of the requirements of software development and operation. For the classical control system in the industrial control area, education is developing slowly. Information experimental teaching mode can get rid of the traditional space, and time constraints, relying on the advantages of computer network. Learners can access the network anytime and anywhere to do experimental teaching work for providing great convenience.

\section{NEW Information technology}

\subsection{Artificial intelligence}

Artificial intelligence as a new method is useful for automatic control. It covers comprehensively computer science, mathematics, information science, cognitive science and so on. Generally speaking, the main goal of artificial intelligence is to try to understand the intelligence essence with the artificial design of computer applications, such that the machines with artificial intelligence may deal with complex tasks. Intelligent technology can improve the stability of electrical automation engineering equipment, and improve the overall quality of electrical automation equipment. Intelligent equipment can ensure the safe operation of electrical equipment, and effectively solve the problems in the operation of electrical equipment. Professional and technical operators must use intelligent technology to effectively control the electrical equipment, which greatly reduces the risk probability of automation equipment, and provides guarantee for the operation stability of the equipment. So in teaching mode, the training of intelligence methods should be necessary. Multilayer neural network [6-7] is a typical tool in artificial intelligence field. The network parameters can be obtained by standard back propagation algorithm. Back propagation is a method to calculate the relationship between the gradient of loss function and model weight in artificial neural network. The weight update of back propagation can be accomplished by a random gradient descent as

$$
w_{i j}(t+1)=w_{i j}(t)+\eta \frac{\partial C}{\partial w_{i j}}+\xi(t)
$$

In Eq.(1), $\eta$ is the learning rate, $\xi(t)$ is the random term, and $C$ is the cost function defined as

$$
C=-\sum d_{j} \log \left(p_{j}\right)
$$

where $d_{j}$ represents the probability of the output $j, p_{j}$ is the probability output of $j$ after activation function, e.g., the Softmax function defined as

$$
p_{j}=\frac{\exp \left(x_{j}\right)}{\sum \exp \left(x_{k}\right)}
$$

where $p_{j}$ represents the probability of the class $j$, and $x_{j}$ and $x_{k}$ represent the inputs of $j$ and $k$.

\subsection{Virtual simulation technology}

Virtual simulation technology [8-9] is to establish a homomorphic simulation model for studying and solving a practical decision-making problem. Virtual simulation modelling mainly includes device modelling, motion modelling, physical modelling, behaviour modelling, etc. Because virtual simulation modelling needs real-time simulation and calculation, it is very different from common data modelling. In addition, virtual simulation also pays attention to many reality details, such as degree of freedom, level of detail model, etc., to make the model move realistically.

In order to improve the real-time calculation efficiency and speed performance of the system, virtual simulation modelling usually focuses on the fidelity of the model. In order to reduce the amount of data, a variety of optimization techniques or strategies can be used. For example, the level of detail technology can divide the scene into some different detail levels according to certain rules. For the current virtual scene, the scene model is called with high level of detail, otherwise the scene model is called with low level of 
detail. In addition, the virtual scene can be divided into several sub scenes according to the modelling needs, and the system can only call the relevant sub scenes to reduce the amount of data. In the field of education and training, virtual reality system has been widely used and developed rapidly in education and scientific research, virtual experiment, security training, etc.

\subsection{Intelligent manufacturing}

Intelligent manufacturing technology is widely used in discrete manufacturing enterprises, and has been widely used in automobile and electronic industry. In the analysis and design of electrical engineering circuit, intelligent technology plays an important role in four aspects: two port network, three-phase circuit, circuit with inductance and periodic non sinusoidal current circuit. Circuit analysis and design requires high accuracy of operation. It is precisely because intelligent technology can automate the computer control function, so through computer control, it can avoid technical errors caused by manual operation and solve the problem that human cannot focus attention, so that the circuit analysis and design in electric and gas engineering is more accurate.

For intelligent manufacturing technology, intelligent factory $[10]$ is constructed with main features: interconnection, optimization, transparency, foresight and agility. These characteristics are helpful for scientific decision-making and help enterprises to improve the production process. Fig.1 shows the system architecture of an intelligent factory.

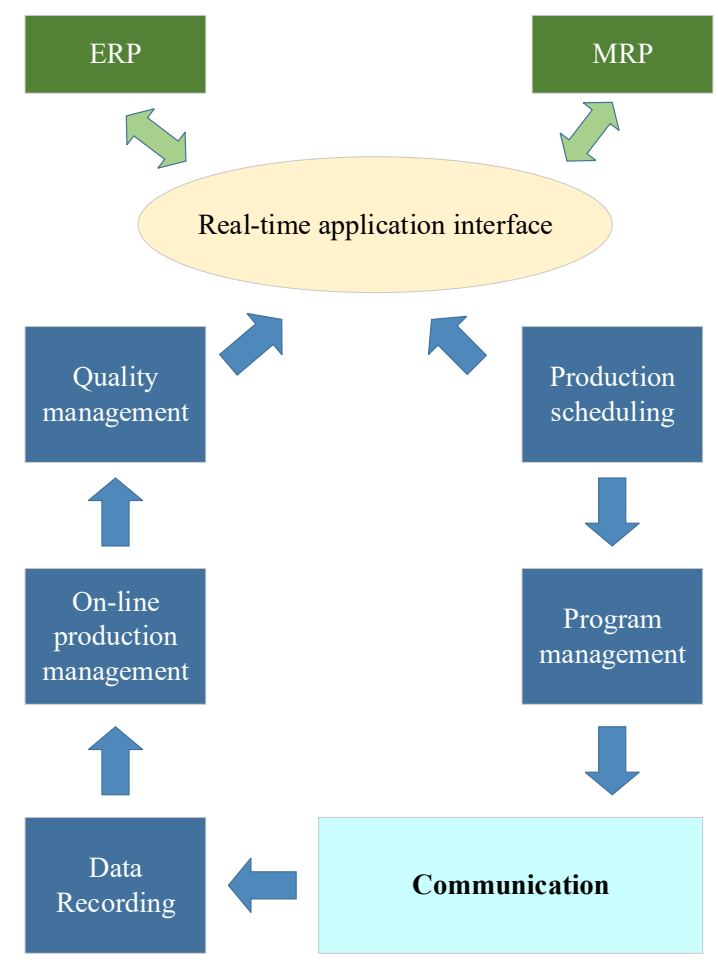

Fig. 1. The system architecture of intelligent manufacturing

Based on a new simulation technology platform, the intelligent manufacturing experiments are developed, which can be used in class experiment of motion control, robot control, industrial network practice, and the design of programmable logic controller technology. More importantly, it can be used as the design and development subject of comprehensive practice of automation specialty. Through these practical teaching technologies, students' autonomous learning ability and engineering practical application ability are cultivated.

\section{Model design}

Virtual simulation technology uses computer programming technology to synthesize a virtual world and it can interact with user sensory feedback with a high degree of real-time simulation. Compared with the traditional forms of content and knowledge, the biggest feature of virtual simulation is that users can interact with each other. Therefore, it can be used as an effective means to enhance the effect of teaching, training and drilling, and solve the high risk and pollution problems and the difficult reproducing problems.

Artificial intelligence technology can provide a new way to deal with unstructured knowledge. It can also provide the corresponding knowledge system according to learners' different knowledge needs. Artificial intelligence technology provide people with efficient and low-cost learning plan. It can analyse learning data, and build expert knowledge and learning base for improving learning efficiency. Electrical automation model based on artificial intelligent and visual simulation technology is established, including system establishment and system development.

In this simulation system, intelligent technology is combined with electrical automation equipment. The application quality and efficiency of automatic control equipment are relatively higher than before. The practical teaching system of virtual reality not only enriches the traditional practical training content, but also combines the advantages and characteristics of virtual teaching.

The practical training and virtual simulation teaching are carried out from theory learning to practice training, and from basic training to promotion training, so as to steadily improve practical ability and innovation ability. The flexible teaching system is adopted to guide the users to improve their innovative consciousness and ability, so that the virtual simulation training can achieve a good effect of helping assistants.

In addition, intelligent technology can realize the control of electrical engineering automation, not only reduces the production cost, but also makes the human resources get reasonable distribution, avoids the excessive waste of resources, reduces the workload of human resource management, and improves the reliability and effectiveness of the operation of electrical engineering automation. The block diagram of virtual simulation system is shown in Fig.2.

The application of intelligent technology in virtual simulation system can truly realize automatic control and management training. In the VR system, a deep neural network regression model is used. When the neural 
network training is finished, the intelligent scoring function can be used to score automatically according to the users' performance in the simulation experiment. The users can complete the experiment operation on the virtual simulation experiment system, and the user's data of the experiment scene will be updated in the system database.

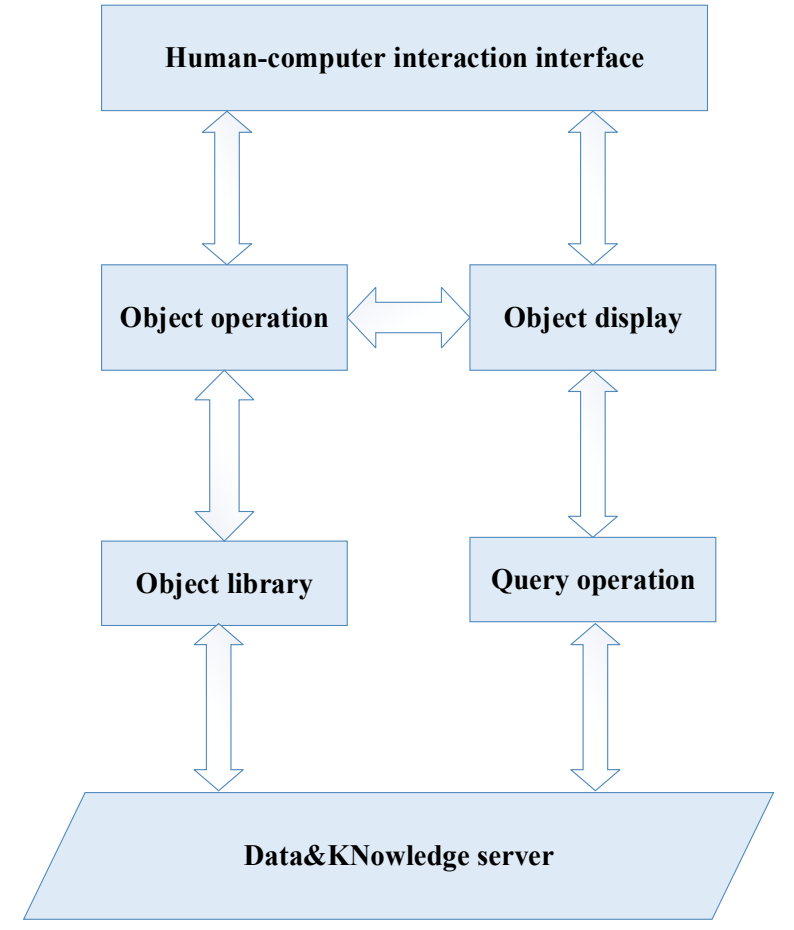

Fig. 2. Block diagram of virtual simulation system

The recording subsystem of the experiment scene completely saves the information of the experimental elements, including measuring and controlling parameters, design processes, and experimental operation information. The detailed data of component parameter, port setting, and connection topology are saved in the dictionary data structure. The feature values are put into the neural network model for calculation, and the prediction ranges for the intelligent scoring model are obtained. With the continuous accumulation of virtual experimental data and the iterative updating of neural network, the intelligent scoring results will accurately reflect the real level of users, and gradually replace the manual marking.

Under the new mode for electrical automation training, due to the introduction of three-dimensional virtual simulation with intelligent technology, students can intuitively understand and learn new knowledge of electrical automation. Meanwhile, the communication ability of the users and their learning initiative can be significantly enhanced.

\section{Conclusion}

Electrical automation technology is an interdisciplinary major involving natural science, technical science and social science. By using the new model, we can take the practical teaching project of electrical automation for intelligent manufacturing specialty as the method reform carrier, as well as the reform of practical teaching system as the breakthrough point of teaching model. In the teaching reform process, there are objective analysis of professional training, class settings, equipment updating, practical teaching system and method research. The teaching mode of electrical automation based on combination of various technologies is still the key work of further study.

\section{Acknowledgments}

This research was supported by the 2021 School Pilot Demonstration Course project in Chongqing University of Posts and Telecommunications (Grant no. XKCSZ2148).

\section{References}

1. Wang G. Research on artificial intelligence technology of electrical automation control. Applied Mechanics and Materials, 624(2014).

2. Huo Y, Ren Y, Feng L, et al. Research on the bilingual education reform in control techniques, 2nd International Workshop on Education Reform and Social Sciences, 2019.

3. Zhang J, Xu XL, Li CC, et al. Study on reform of innovative teaching curriculum system on major of environmental engineering, The International Conference on Industrial Engineering and Manufacturing Technology, 2014.

4. Gainor S J, Goins R T, Miller L A . Using online modules is a multi-modality teaching system: a high-touch high-tech approach to geriatric education. Gerontology \& Geriatrics Education, 24(2004).

5. Gan B. Design and application research of VR/AR teaching experience system. Journal of Physics: Conference Series, 1187(2019).

6. Krawczak M. Multilayer Neural Networks: A Generalized Net Perspective, Springer, Heidelberg, 2004.

7. Brightwell G, Kenyon C, Paugam-Moisy H . Multilayer Neural Networks. Journal of Indian Institute of Science, 1(2003).

8. Taxén G, Naeve A. A system for exploring open issues in VR-based education. Computers \& Graphics, 26(2002).

9. Manseur R. Virtual reality in science and engineering education, 35th Annual Conference of Frontiers in Education, 2005.

10. Maruyama Y, Yagi J, Takada H, et al. Development of virtual and real-field construction management system for innovative, intelligent field factorydevelopment of virtual construction field. Isarc Proceedings, 2000. 